\title{
X-ray studies of enzymes that interact with penicillins
}

\author{
J. A. Kelly ${ }^{a}$, A. P. Kuzin ${ }^{a}$, P. Charlier ${ }^{b, *}$ and E. Fonzé ${ }^{b}$ \\ a Department of Molecular and Cell Biology, Institute of Materials Science, University of Connecticut, Storrs, \\ Connecticut (USA) \\ ${ }^{\mathrm{b}}$ Centre d'Ingénierie des Protéines, Unité de Cristallographie, Institut de Physique, Université de Liège, \\ Sart Tilman, B-4000 Liège (Belgium), Fax +32 4366 4741, e-mail: paulette.charlier@ulg.ac.be
}

\begin{abstract}
The technique of X-ray diffraction has been successfully applied to enzymes associated with peptidoglycan biosynthesis. The technique has taught us a great
\end{abstract}

deal about the structures and catalytic mechanisms of penicillin-binding proteins and $\beta$-lactamases. An insight into the structural basis for antibiotic resistance is given.

Key words. X-ray diffraction; $\beta$-lactamases; peptidases; penicillin-binding proteins; active-site serine.

\section{Introduction}

Over the last two decades, biophysical techniques have become increasingly important in the study of proteins. One extremely powerful method for investigating protein structure and function is X-ray diffraction. This is the case despite the fact that there are serious hurdles that one must overcome to realize the rich results that can be obtained from the X-ray approach. Chief among the difficulties is the need for significant quantities of pure protein sample. JeanMarie Ghuysen and his colleagues at the University of Liège have made extraordinary contributions to our understanding of the nature of bacterial cell walls and the enzymes associated with the peptidoglycan biosynthesis. They have also been responsible for the elegant characterization and purification work that has made available for biophysical studies many of the penicillin-binding proteins (PBPs) and $\beta$-lactamases that are key components of cell wall biochemistry [1].

Penicillin binding proteins are enzymes involved in the final stages of bacterial cell wall synthesis. These enzymes present a wide range of molecular weights from

* Corresponding author.
27 to $120 \mathrm{kDa}$. The largest of these enzymes are bifunctional enzymes with a domain responsible for transglycosylation in the nascent glycan strands and a domain associated with the cross-linking of the peptide portions of the cell wall. The low molecular weight PBPs are strictly D-alanyl-D-alanine peptidases. The DD-peptidases can act as carboxypeptidases and transpeptidases, catalysing the scission of the terminal D-alanyl-D-alanine bond in the peptide portion of the growing cell wall and the subsequent formation of a peptide bridge to an appropriate amino acceptor on an adjacent peptidoglycan strand. There are, however, examples of DD-peptidases that function solely or primarily as DD-carboxypeptidases, limiting their reaction to cleavage of the D-alanyl-D-alanine bond (e.g. the DD-carboxypeptidase from Bacillus stearothermophilus [2]). There are also DD-peptidases that are characterized as strict DD-transpeptidases such as the enzyme from Streptomyces K15 [3]. Penicillin-binding proteins are the targets in bacteria for $\beta$-lactam antibiotics (penicillins and cephalosporins). These drugs serve as potent antibacterial agents because they inhibit the PBPs in the growing bacteria, preventing the crucial cross-linking of the cell wall. Their effectiveness is the result of the structural analogy of the drugs to the normal D-alanyl-D-alanine peptide substrates of the PBPs. 
Table 1. Penicillin-interacting enzymes whose structures have been determined.

\begin{tabular}{|c|c|c|c|c|c|c|}
\hline Enzyme & Source & Catalytic entity & MW & $\begin{array}{l}\text { PDB* } \\
\text { code }\end{array}$ & Resolution & Reference \\
\hline \multirow[t]{4}{*}{ PBP } & S. albus $\mathrm{G}$ & Zinc & 18,000 & $1 \mathrm{LBU}$ & $1.8 \AA$ & Wery, D. (1982) Nature 299: 5882, 469-470 \\
\hline & S. K15 & Serine & 27,474 & 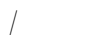 & $2.0 \AA ̊$ & Fonzé, C. (1997) unpublished \\
\hline & S. pneumoniae $\mathrm{PBP} 2 \mathrm{x}$ & Serine & 77,059 & 1PMD & $3.5 \AA$ & Pares, D. (1996) Nature Stuct. Biol. 3: 284-289 \\
\hline & $S$. R61 & Serine & 37,500 & 3PTE & $1.6 \AA ̊$ & Kelly (1995) J. Mol. Biol. 254: 223-236 \\
\hline \multirow[t]{13}{*}{$\beta$-Lactamase } & B. cereus $569 / \mathrm{H}$ & Class B zinc & 26,000 & 1BME & $1.85 \AA$ & Carfi, D. (1995) EMBO J. 14: 20, 4919-4921 \\
\hline & B. fragilis & Class B zinc & 26,000 & $1 \mathrm{ZNB}$ & $1.85 \AA$ & Concha, H. (1996) Structure 4: 7, 823-836 \\
\hline & & & & 1BMI & $2.0 \AA$ & Carfi, D. (1997) Acta Cryst. D53: 485-487 \\
\hline & B. cereus $5 / \mathrm{B} / 6$ & Class B zinc & 26,000 & 1 & $1.9 \AA$ & Fonzé, C. (1997) unpublished \\
\hline & S. albus $\mathrm{G}$ & Class A Serine & 28,500 & 1 & $1.7 \AA ̊$ & Dideberg, C. (1987) Biochem. J. 245: 911-913 \\
\hline & B. licheniformis & Class A Serine & 29,500 & 4BLM & $2.0 \AA$ & Moews, K. (1991) J. Mol. Biol. 220: 435-455 \\
\hline & S. aureus & Class A Serine & 28,500 & 3BML & $2.0 \AA$ & Herzberg (1991) J. Miol. Biol. 217: 701-719 \\
\hline & E. coli TEM1 & Class A Serine & 28,950 & / & $1.7 \AA$ & Strynadka, J. (1992) Nature 359: 700-705 \\
\hline & & & & 1BTL & $1.8 \AA ̊$ & Jelsch, S. (1993) Proteins 16: $364-383$ \\
\hline & & & & $1 \mathrm{XPB}$ & $1.9 \AA$ & Fonzé, C. (1995) Acta Cryst. D51: 682-694 \\
\hline & C. freundii & Class C Serine & 39,000 & & $2.0 \AA$ & Oefner, W. (1990) Nature 343: 284-288 \\
\hline & E. cloacae $\mathrm{P} 99$ & Class C Serine & 39,000 & $2 \mathrm{BLT}$ & $2.0 \AA$ & Lobkovsky, K. (1993) PNAS 90: 11257-11262 \\
\hline & E. cloacae $908 \mathrm{R}$ & Class C Serine & 39,000 & 1 & $2.5 \AA$ & Fonzé, C. (1997) unpublished \\
\hline
\end{tabular}

*Protein Data Bank.

A second important class of enzymes that affects cell wall biosynthesis is that of the $\beta$-lactamases. These enzymes are believed to have evolved from the cell wall-synthesizing PBPs but with an important change in their catalytic mechanism. Instead of being inhibited by $\beta$-lactams, $\beta$-lactamases rapidly hydrolyse the $\beta$-lactam bond and release the inactive reaction product, thereby protecting the cell from the action of the drugs. $\beta$-Lactamases are grouped into four classes, A, B, C and D. Classes A, C and D rely on a reactive serine for catalysis, as do most PBPs. Their classification is based on amino acid sequence. Class B $\beta$-lactamases are metalloenzymes that require zinc ions for activity.

\section{X-ray diffraction studies}

To date, X-ray diffraction has been carried out on 4 PBPs and $10 \beta$-lactamases (see table 1) with many additional examples of complexes or mutants forms of the enzymes. A crucial problem in X-ray diffraction studies is the loss of phase information during the diffraction experiment. The phase problem has been solved primarily by multiple isomorphous replacement using heavy atom derivatives of the native enzymes (e.g. Streptomyces R61 DD-peptidase [4]), and more recently by molecular replacement using previously determined protein structures as search models (e.g. the TEM1 $\beta$-lactamase [5]). The biggest limitation of molecular replacement in the case of enzymes that exhibit a wide variation in their amino acid sequences is the low similarity with the probe structure. Up to now, only a few enzymes interacting with penicillin have been solved this way. However, with the increasing number of independently solved structures, we may expect more extensive use of this method.
The DD-peptidase domains of the bifunctional PBPs and of most monofunctional PBPs, as well as the majority of the $\beta$-lactamases known today, are serine enzymes that form acyl intermediates. Amino acid sequence similarity between the different classes of PBPs (bi- and monofunctional) and between the three classes of serine- $\beta$-lactamases (A, C and D) is as low as between PBPs and $\beta$-lactamases, usually in the range of 10 to $15 \%$. Among members of a given class, however, the similarity may range from more than $90 \%$ for the class C $\beta$-lactamases to $50 \%$ for the class A $\beta$-lactamases. It is interesting to note that for the $S$. R61 DD-peptidase, the highest primary sequence homology is found with the class $\mathrm{C} \beta$-lactamases (about 25 to $28 \%$ ).

We have learned a great deal about these enzymes through structural studies. Both serine-mediated PBPs and $\beta$-lactamases share an overall two-domain structure. One domain is all helical, and the second contains a $\beta$-sheet flanked by helices on both faces of the sheet (fig. $1 \mathrm{a}-\mathrm{d}$ ). The reactive serine is found at the interface of these two domains. There is higher tertiary structural resemblance between the $S$. R61 DD-peptidase and the class $\mathrm{C} \beta$-lactamases (as expected from the size of the molecules and their sequence similarity), and between the $S$. K15 DD-transpeptidase and the class A $\beta$-lactamases (even though it was not evident from the poor sequence similarity of $15 \%$ ), than between the class A $\beta$-lactamases and the class $\mathrm{C} \beta$-lactamases.

There are only three brief sequence segments common to all PBPs and $\beta$-lactamases, each a key element of the active site (fig. 2a,b). These include SXXK, with the reactive serine followed by two variable residues and then an absolutely conserved lysine. This motif is found 


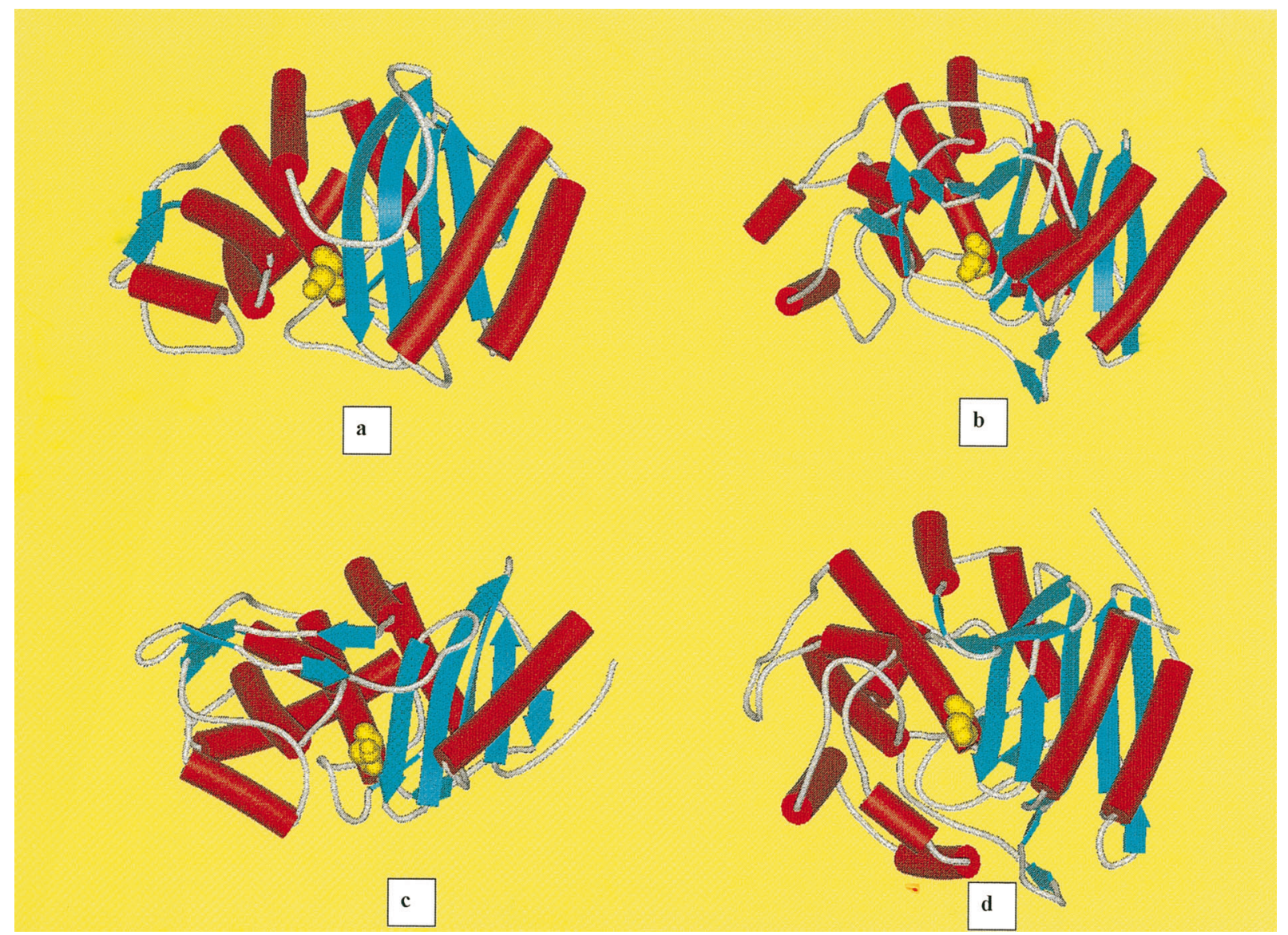

Figure 1. Schematic drawings of the class A $\beta$-lactamase of E. coli TEM1 (a), class C $\beta$-lactamase of Enterobacter cloacae 908R (b), DD-transpeptidase/PBP of Streptomyces K15 (c) and DD-carboxypeptidase-transpeptidase/PBP of Streptomyces R61 (d). The $\beta$-strands are drawn as blue arrows and $\alpha$-helices as red cylinders. The reactive serine atoms are drawn as yellow spheres, at the amino-terminus of the central helix $\alpha 2$.

at the amino terminus of a helix generally within 70 residues of the amino terminus of the low molecular weight PBPs and the $\beta$-lactamases. The second region is a triad found on a loop in the active site below the reactive serine. The $\mathrm{SDN}$ motif in class A $\beta$-lactamases is replaced by SGC in the $S$. K15 DD-transpeptidase, and by YXN in the $S$. R61 DD-peptidase and the class C $\beta$-lactamases. The hydroxyl group of the tyrosine in these enzymes is in the same position as the hydroxyl of the serine in the SDN enzymes. The last conserved region is $\mathrm{KT} / \mathrm{SG}$ in all enzymes except the $S$. R61 DD-peptidase, which has an HTG triad. This motif is found on the innermost $\beta 3$ strand of the $\beta$-sheet, forming one edge of the active site pocket. It is this motif that orients the incoming peptide substrate or $\beta$-lactams, binding them as though they were extensions of the $\beta$-sheet of the protein. The class A $\beta$-lactamases have an additional active-site-defining motif EXELN, located at the entrance of the cavity near the bottom of the $\beta 3$ strand. This motif defines the so-called $\Omega$-loop and contains the essential residue Glu 166. This Glu 166, with Asn 170, positions a strictly conserved structural water molecule, thus playing an important role in the catalysis of penicillin hydrolysis by class A $\beta$-lactamases. In the case of the $S$. K15 dd-transpeptidase, a loop of different shape is positioned in the same direction, but the possible acidic equivalent to Glu 166 is pointing away from the active site. A similar loop may be observed in the $S$. R61 DD-peptidase and the class C $\beta$-lactamases, but the peptide chain is in the opposite sense, and there is no possible structural equivalent to Glu 166. Compared with the $S$. K15 DD-transpeptidase and the class A $\beta$-lactamases, the $S$. R61 DD-peptidase and the class $\mathrm{C} \beta$-lactamases have additional loops and secondary structure elements away from the active site. Several crystallographic binding studies have been performed on these enzymes. In the case of the the class A $\beta$-lactamases, the first reported complex was the TEM Glu166Asn mutant acylated by penicillin G [6]. Complexes of the Staphylococcus aureus lactamase with clavulanate (PDB entry code 1BLC) and a methylphosphonate monoester monoanion inhibitor (PDB entry code $1 \mathrm{BLH}$ ) have been also studied at liquid nitrogen and room temperature, respectively [7, 8]. More recently, two novel designed inhibitors have been used for crystallographic binding studies on the TEM enzyme, 
(a)

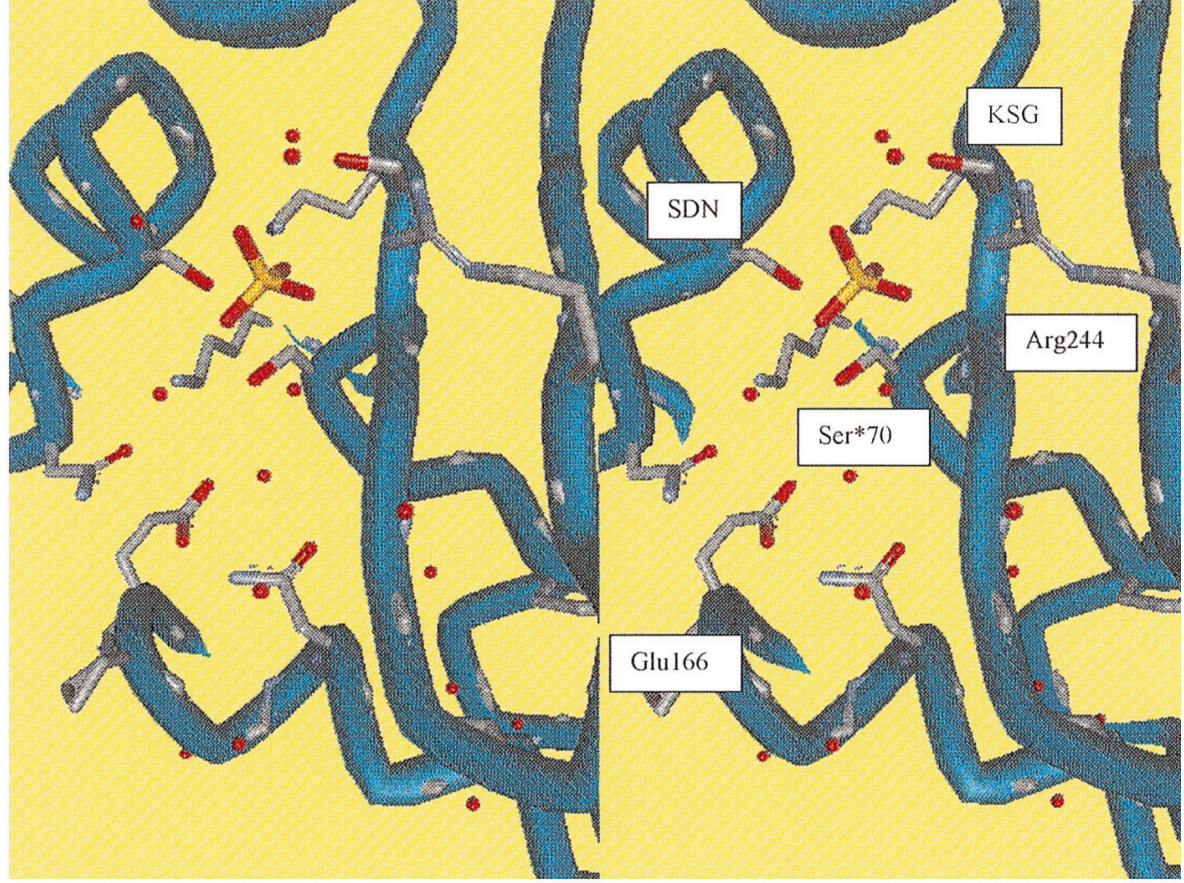

(b)

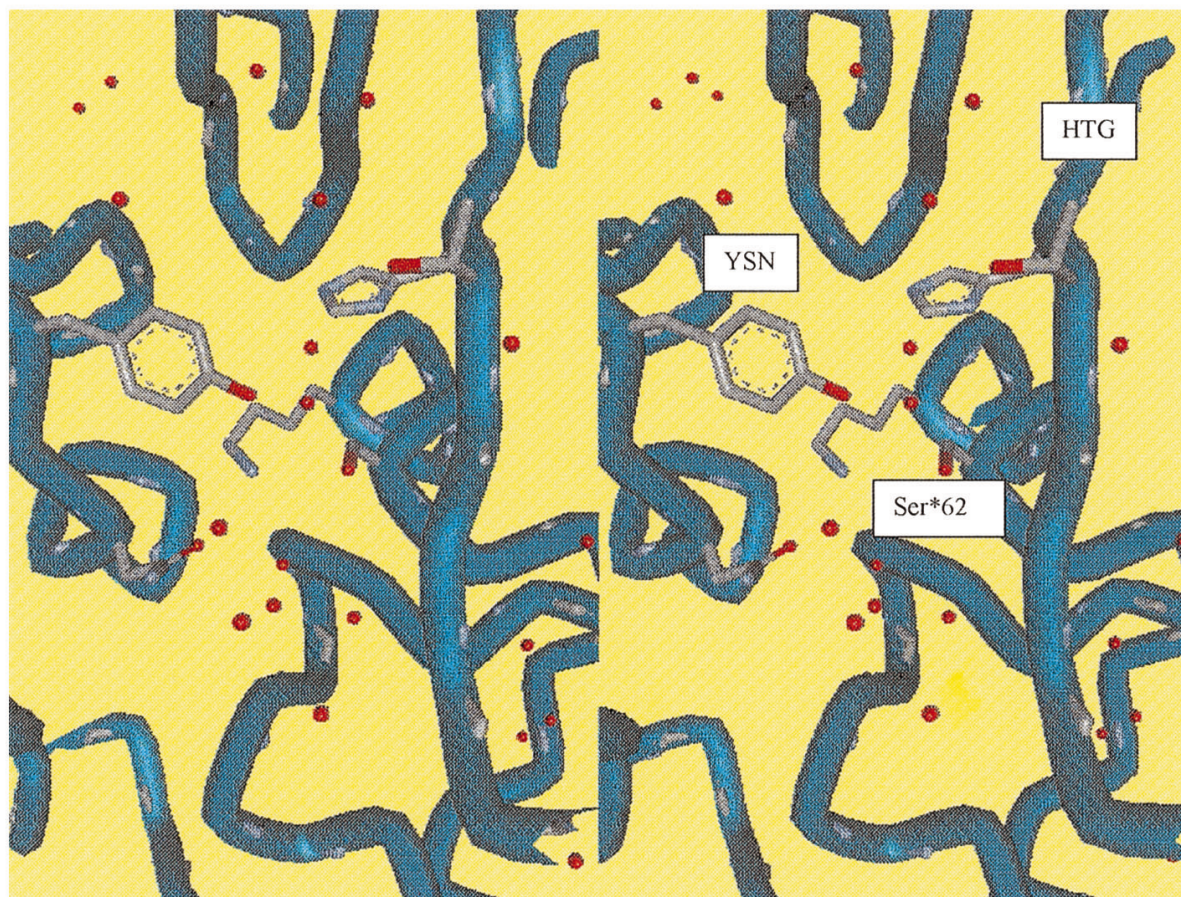

Figure 2. Stereoviews of the catalytic cleft of the class A $\beta$-lactamase of E. coli TEM1 (a) and the DD-carboxypeptidase-transpeptidase/ PBP of Streptomyces R61 (b), including the conserved active-site groups Ser70, Lys73, Ser130, Asn132, Glu166, Asn170, Lys234, Ser235 and Arg244 for the class A $\beta$-lactamase of E. coli TEM1, and Ser62, Lys65, Tyr159, Asn161, His298 and Thr299 for the DD-carboxypeptidase-transpeptidase/PBP of Streptomyces R61. The structural water molecules are drawn as small red spheres. One structural $\mathrm{SO}_{4}$ ion is drawn in yellow in $(a)$.

the $6 \alpha$-(hydroxymethyl)penicillanate (PDB entry code 1TEM) [9] and the $(1 R)-1$-acetamido-2-(3-carboxyphenyl)ethane boronic acid [10]. Two complexed class $\mathrm{C}$ $\beta$-lactamase structures have been reported in the literature, the Citrobacter freundii enzyme complexed with the monobactam inhibitor aztreonam [11] and the Enterobacter cloacae enzyme complexed with a phosphonate derivative (PDB entry code 1BLS) [12].

For the DD-peptidases, binding of the $S$. R61 enzyme with cephalosporins has been studied by X-ray crystal- 


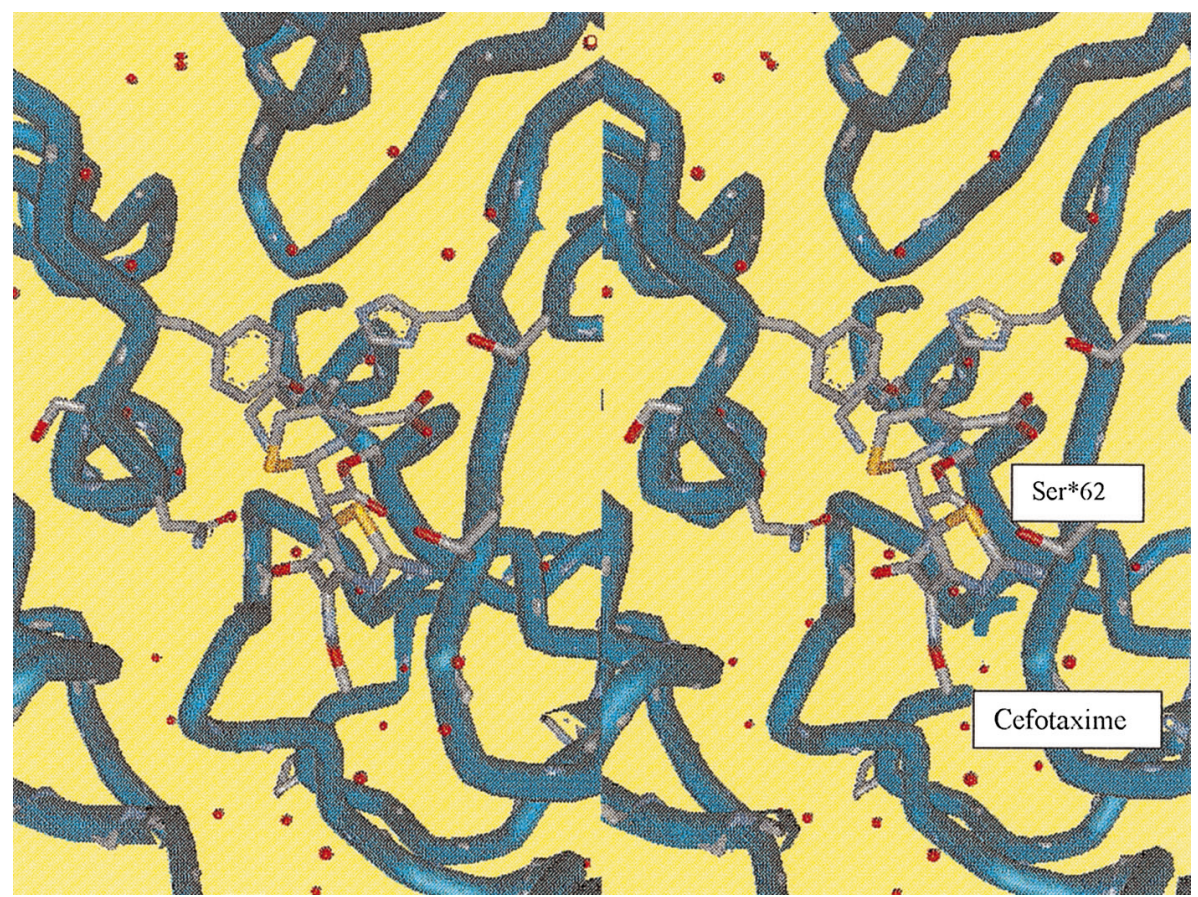

Figure 3. Stereoview of the catalytic cleft of the the DD-carboxypeptidase-transpeptidase/PBP of Streptomyces R61 complexed with cefotaxime, showing the the $\beta$-lactam antibiotic bound to the reactive serine Ser62.

lography [13]. This enzyme exhibits a remarkable stability with the cephalosporinyl intermediates. This stability is said to be the result of the lack of a well-positioned water molecule in the active site that is observed in the $\beta$-lactamase structures. In the class $\mathrm{A} \beta$-lactamases, this water molecule is thought to be activated by active site residues for nucleophilic attack on the acyl bond. Its absence would be consistent with the failure of the $S$. R61 DD-peptidase to deacylate $\beta$-lactam intermediates, which leads to their efficacy as antibiotics. Another interesting observation made on the structure with cefotaxime, a clinically important $\beta$-lactam, is the conformational change in the side chain of Thr 301 (as shown in fig. 3). In all other $\beta$-lactamoyl and phosphonyl complexes studied, a hydrogen bond is made between the backbone of this residue or its equivalent and the drug side chain. The bulky, rigid oxime side chain of cefotaxime prevents this main-chain hydrogen bond from being formed. A change in the orientation of the threonine side chain positions the side chain hydroxyl group so that it can hydogen bond to the drug molecule. A cefotaxime-resistant laboratory mutant of Streptococcus pneumoniae has been isolated that is characterized by a Thr to Ala mutation at the equivalent site [14]. Such a mutation would not allow the substitution of a side-chain hydrogen bond for the lost main-chain hydrogen bond. It is clear that structural studies of these penicillin-interacting enzymes have been and will continue to be of significant importance for understanding the emerging resistance of the PBPs and $\beta$-lacta- mases to the third-generation $\beta$-lactam antibiotic such as cefotaxime.

\section{Conclusions}

X-ray studies have demonstrated that the serine $\beta$-lactamases and PBPs are structurally related and that they must have evolved (and are still evolving) from a common ancestor with preservation of much of the same serine-assisted acyl transfer machinery. However, the question of which structural features determine the different functions, such as peptide bond transfer or hydrolysis and penicillin binding or hydrolysis, remains unclear.

Acknowledgement. The authors acknowledge the extensive work of our colleagues O. Dideberg, J. R. Knox and P. C. Moews on PBPs and $\beta$-lactamases. The work in Liège was supported by the Belgian programme on Interuniversity Poles of Attraction initiated by the Belgian State, Prime Minister's Office, Services fédéraux des affaires scientifiques, techniques et culturelles (PAI no. $\mathrm{P} 4 / 03)$.

1 Ghuysen J.-M. (1991) Serine $\beta$-lactamases and penicillin-binding proteins. Ann. Rev. Microb. 45: 37-65

2 Despreaux C. W. and Manning R. F. (1993) The dac A gene of Bacillus stearothermophilus coding for D-alanine carboxypeptidase: cloning, structure and expression in E. coli and Pichia pastoris. Gene 131: 35-41

3 Nguyen-Distèche M., Leyh-Bouille M., Pirlot S., Frère J.-M. and Ghuysen J.-M. (1986) Streptomyces K15 DD-peptidasecatalysed reactions with ester and amide carbonyl donors. Biochem. J. 235: 167-176 
4 Kelly J. A. and Kuzin A. P. (1995) The refined crystallographic structure of a DD-peptidase penicillin-target enzyme at 1.6 Å resolution. J. Mol. Biol. 254: 223-236

5 Fonzé E., Charlier P., Tóth Y., Vermeire M., Raquet X. and Frère J.-M. (1995) TEM1 $\beta$-lactamase structure solved by molecular replacement and refined structure of the S235A mutant. Acta Cryst. D51: 682-694

6 Strynadka N. C. J., Adachi H., Jensen S. E., Johns H., Sielecki A., Betzel C. et al. (1992) Molecular structure of the acyl-enzyme intermediate in $\beta$-lactam hydrolysis at enzyme at $1.7 \AA$ resolution. Nature (Lond.) 359: 700-705

7 Chen C. C. H. and Herzberg O. (1992) Inhibition of $\beta$-lactamases by clavulanate. Trapped intermediates in cryocristallographic studies. J. Mol. Biol. 224: 1103-1113

8 Chen C. C. H., Rahil J., Pratt R. F. and Herzberg O. (1993) Structure of a phosphonate-inhibited $\beta$-lactamase: an analog of the tetrahedral transition state/intermediate of $\beta$-lactam hydrolysis. J. Mol. Biol. 234: 165-178

9 Maveyraud L., Massova I., Birck C., Miyashita K., Samama J.-P. and Mobashery S. (1996) Crystal structure of a $6 \alpha$-(hydroxymethyl)penicillanate complexed to the TEM1 $\beta$-lactamase from E. coli: evidence on the mechanism of action of a novl inhibitor designed by a computer-aided process. J. Am. Chem. Soc. 118: $7435-7440$
10 Strynadka N. C. J., Martin R., Jensen S. E, Gold M. and Jones J. B. (1996) Structure-based design of a potent transition state analogue for the TEM-1 $\beta$-lactamase. Nature Struct. Biol. 3: 688-695

11 Oefner C., D’Arcy A., Daly J. J., Gubernator K., Charnas R. L., Heinze I. et al. (1990) Refined crystal structure of $\beta$-lactamase from Citrobacter freundii indicates a mechanism for $\beta$-lactam hydrolysis. Nature 343: $284-288$

12 Lobkovsky E., Billings E. M., Moews P. C., Rahil J., Pratt R. F. and Knox J. R. (1994) Crystallographic structure of a phosphonate derivative of the Enterobacter cloacae $P 99$ cephalosporinase: mechanistic interpretation of a $\beta$-lactamase transition state analog. Biochemistry 33: 6762-6772

13 Kuzin A. P., Liu H., Kelly J. A. and Knox J. R. (1995) Binding of cephalothin and cefotaxime to D-Ala-D-Ala peptidase reveals a functional basis of a natural mutation in a low-affinity penicillin-binding protein and in extended-spectrum $\beta$-lactamases. Biochemistry 34: $9532-9540$

14 Coffey T. J., Daniels M., McDougal L. K., Dowson C. G., Tenover F. C. and Spratt B. G. (1995) Genetic analysis of clinical isolates of Streptococcus pneumoniae with high-level resistance to expanded-spectrum cephalosporins. Antimicrob. Agents Chemother. 39: 1306-1313 